\title{
RESEARCH
}

Open Access

\section{A NOTCH1/LSD1/BMP2 co-regulatory network mediated by miR-137 negatively regulates osteogenesis of human adipose- derived stem cells}

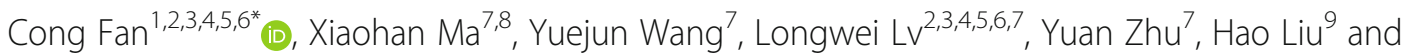
Yunsong Liu $\mathrm{Li}^{7,2,3,4,5,6}$

\begin{abstract}
Background: MicroRNAs have been recognized as critical regulators for the osteoblastic lineage differentiation of human adipose-derived stem cells (hASCs). Previously, we have displayed that silencing of miR-137 enhances the osteoblastic differentiation potential of hASCs partly through the coordination of lysine-specific histone demethylase 1 (LSD1), bone morphogenetic protein 2 (BMP2), and mothers against decapentaplegic homolog 4 (SMAD4). However, still numerous molecules involved in the osteogenic regulation of miR-137 remain unknown. This study aimed to further elucidate the epigenetic mechanisms of miR-137 on the osteogenic differentiation of hASCs.

Methods: Dual-luciferase reporter assay was performed to validate the binding to the $3^{\prime}$ untranslated region ( $3^{\prime}$ UTR) of NOTCH1 by miR-137. To further identify the role of NOTCH1 in miR-137-modulated osteogenesis, tangeretin (an inhibitor of NOTCH1) was applied to treat hASCs which were transfected with miR-137 knockdown lentiviruses, then together with negative control (NC), miR-137 overexpression and miR-137 knockdown groups, the osteogenic capacity and possible downstream signals were examined. Interrelationships between signaling pathways of NOTCH1-hairy and enhancer of split 1 (HES1), LSD1 and BMP2-SMADs were thoroughly investigated with separate knockdown of NOTCH1, LSD1, BMP2, and HES1.

Results: We confirmed that miR-137 directly targeted the 3' UTR of NOTCH1 while positively regulated HES1. Tangeretin reversed the effects of miR-137 knockdown on osteogenic promotion and downstream genes expression. After knocking down NOTCH1 or BMP2 individually, we found that these two signals formed a positive feedback loop as well as activated LSD1 and HES1. In addition, LSD1 knockdown induced NOTCH1 expression while suppressed HES1.

Conclusions: Collectively, we proposed a NOTCH1/LSD1/BMP2 co-regulatory signaling network to elucidate the modulation of miR-137 on the osteoblastic differentiation of hASCs, thus providing mechanism-based rationale for miRNA-targeted therapy of bone defect.
\end{abstract}

Keywords: MicroRNA, Human adipose-derived stem cells, Osteogenesis, NOTCH1, Signaling

\footnotetext{
* Correspondence: congcongsherry@sina.com

'Department of General Dentistry II, Peking University School and Hospital of

Stomatology, Beijing, China

${ }^{2}$ National Center of Stomatology, Beijing, China

Full list of author information is available at the end of the article
}

\section{$\triangle B M C$}

(c) The Author(s). 2021 Open Access This article is licensed under a Creative Commons Attribution 4.0 International License, which permits use, sharing, adaptation, distribution and reproduction in any medium or format, as long as you give appropriate credit to the original author(s) and the source, provide a link to the Creative Commons licence, and indicate if changes were made. The images or other third party material in this article are included in the article's Creative Commons licence, unless indicated otherwise in a credit line to the material. If material is not included in the article's Creative Commons licence and your intended use is not permitted by statutory regulation or exceeds the permitted use, you will need to obtain permission directly from the copyright holder. To view a copy of this licence, visit http://creativecommons.org/licenses/by/4.0/. The Creative Commons Public Domain Dedication waiver (http://creativecommons.org/publicdomain/zero/1.0/) applies to the data made available in this article, unless otherwise stated in a credit line to the data. 


\section{Background}

As we know, human adipose-derived stem cells (hASCs) have multilineage differentiation potentials and good application prospects for bone regeneration [1-3] because of their abundant sources and easy access for clinical uses [4]. Though plenty of signaling pathways, such as Wnt/ $\beta$-catenin signal [5], glycogen synthase kinase 3 signal [6], bone morphogenetic protein (BMP) signal [7-9], NOTCH signal [10, 11], and extracellular regulated protein kinases signal $[7,12,13]$ have been demonstrated to dominate osteoblastic differentiation of hASCs, considerable research is still necessary to reveal intricate interactions between various signals and make further advances in hASC-based cell therapy.

MicroRNAs (MiRNAs) are a class of endogenous and highly conserved small non-coding single-stranded RNAs, which mediate post-transcriptional gene regulation [14] and play crucial roles in the proliferation and osteogenic differentiation of hASCs [5, 10, 15-18]. MiR-137 has manifested its anti-tumor activity in multiple malignancies, such as glioblastoma [19], melanoma [20], multiple myeloma [21], non-small cell lung cancer [22], and endometrial cancer [23]. In neural development, several studies confirm that miR-137 regulates cell proliferation, differentiation [24-28], and neuronal maturation [29-31] in adult or mouse stem cells. Nevertheless, during the process of hASCs differentiating into osteoblastic lineage, the function and epigenetic mechanisms of miR-137 have not been investigated except for our previous study [32], in which we disclose part of the mechanisms as the coordination between lysine-specific histone demethylase 1 (LSD1) and BMP2-mothers against decapentaplegic homolog 4 (SMAD4) pathway. Considering that the relationships of osteogenesis-associated signals are complex and diverse, and massive molecules participating in the $L S D 1 / B M P 2 /$ $S M A D 4$ network remain unascertained, we need to further clarify the regulatory mechanisms of miR-137 on the osteogenesis.

$\mathrm{NOTCH}$ signal is a fundamental pathway in bone remodeling and skeletal homeostasis [33-35]. Hairy and enhancer of split 1 (HES1), a downstream gene of $\mathrm{NOTCH}$ [36], is responsible for the actions of NOTCH in the skeleton, even though its osteogenic effects are cell type-specific and context-dependent. By interacting with runt-related transcription factor 2 (RUNX2), HES1 induces osteopontin promoter [37]. But HES1 also binds to the osteocalcin $(O C N)$ promoter and suppresses its transcription in osteoblastic cells [38]. HES1 inactivation not only increases the femoral length and trabecular number in the limb bud of transgenic mice, but also enhances mineral apposition rate and suppresses bone resorption [39]. NOTCH1 has emerged as a target of miR137 in human renal mesangial cells [40], retinal ganglion cells [41], neurons [42], non-small cell lung cancer cells
[43], and breast cancer cells [44], but whether it is directly inhibited by miR-137 has not yet been identified in hASCs. In small cell lung cancer cells, NOTCH1 pathway is activated by $L S D 1$ inhibitor and suppressed due to the binding of LSD1 [45]. Additionally, the induction of $N O T C H$ signal impairs the activation of $B M P$ pathway and the osteoblastic differentiation of dental follicle cells [46]. In contrast, NOTCH1 upregulates BMP2 expression in human aortic valve interstitial cells through the stimulation of $N F-K B$ [47]. Our previous study confirmed that miR-137 knockdown induced BMP2-SMAD4 pathway through the downregulation of $L S D 1$ dependently or independently [32], which coincides with the studies stating that LSD1 inhibition leads to increased BMP2 expression $[48,49]$. Accordingly, we postulate a signaling network entailing NOTCH1-HES1, LSD1, and BMP2SMADs pathways to unveil the miR-137 modulation on the osteogenesis of hASCs.

This study identified the interactions of miR-137 and its downstream genes and revealed that the coregulatory signaling network of NOTCH1/LSD1/BMP2 mediated by miR-137 negatively modulated the osteogenesis of hASCs, suggesting that miR-137 might be applied as a promising therapeutic target for bone regeneration.

\section{Methods \\ Mice}

The animal experiments were conducted in strict conformity with the guidelines of Animal Welfare Committee of Health Science Center in Peking University (LA2019019). Male, 5-week-old BALB/c-nu/nu nude mice (Charles River, Wilmington, MA, USA) were randomly assigned to 3 groups ( $n=6$ per group) and maintained with specific pathogen-free conditions.

\section{Cell lines}

The hASCs isolated from three separate donors were purchased in ScienCell Research Laboratories (Carlsbad, CA, USA). For each donor, the in vitro cell experiments were performed at least three times individually. For proliferation culture, cells were maintained in proliferation medium (PM), containing Dulbecco's modified Eagle's medium (Thermo Fisher Scientific, Rockford, IL, USA), $1 \%(\mathrm{v} / \mathrm{v})$ penicillin/streptomycin (Thermo Fisher Scientific) and 10\% (v/v) fetal bovine serum (ExCell Bio, Shanghai, China). When the cells reached $70-80 \%$ confluence, osteoinduction was performed by adding osteogenic medium (OM), which contained the above culture medium for promoting proliferation, $100 \mathrm{nM}$ dexamethasone (Sigma-Aldrich), $0.2 \mathrm{mM}$ L-ascorbic acid (Sigma-Aldrich), and $10 \mathrm{mM} \beta$-glycerophosphate (Sigma-Aldrich). The cell culture conditions were $37^{\circ} \mathrm{C}$ with $5 \% \mathrm{CO}_{2}$ and $100 \%$ relative humidity. 


\section{Lentivirus transfection}

Recombinant lentiviruses carrying green fluorescent protein (GFP)-tagged plasmid vectors of negative control (NC), miR-137 overexpression (miR-137), miR-137 knockdown (anti-miR-137), NOTCH1 shRNA (anti-NOTCH1), LSD1 shRNA (anti-LSD1), BMP2 shRNA (anti-BMP2), and HES1 shRNA (anti-HES1) (Additional file 5: Table S1) were produced and packaged by GenePharma (Suzhou, Jiangsu, China). Lentiviral transfection of hASCs were conducted at a multiplicity of infection of 100 for $24 \mathrm{~h}$ with the presence of $5 \mathrm{mg} / \mathrm{mL}$ polybrene, and then selected by $1 \mu \mathrm{g} / \mathrm{ml}$ puromycin (Sigma-Aldrich). The transfection rates of lentiviruses were estimated by counting the number of GFP-tagged cells and total cells with an inverted fluorescence microscope (TE2000-U, Nikon, Tokyo, Japan). The hASCs transfected with miR-137 knockdown lentiviruses were seeded in 96-well plates $(1 \times$ $10^{4}$ cells/well) and treated with tangeretin (an inhibitor of NOTCH1; APExBIO, Houston, TX, USA) at a concentration of $5 \mu \mathrm{M}$ before the examination of osteogenic differentiation ability and downstream gene expression.

\section{Alkaline phosphatase (ALP) staining and quantification} After 7 days of culture in PM or OM, the hASCs were used for ALP staining and activity test according to the published protocol [10]. ALP staining was operated following the BCIP/NBT staining kit (Beyotime, Shanghai, China) instructions. For the quantitative tests of ALP activities, cells were washed with phosphate buffer saline (PBS) and 1\% Triton X-100 (Solarbio, Beijing, China), then scraped in milli- $\mathrm{Q}$ water and subjected to three cycles of freezing and thawing. By employing the BCA method and the pierce BCA protein assay kit (Thermo Fisher Scientific), total protein was read at $562 \mathrm{~nm}$ and computed with a bovine serum albumin standard curve according to the manufacturer's protocol. Afterwards, ALP activity was detected at $520 \mathrm{~nm}$ applying an alkaline phosphatase assay kit (Jiancheng, Nanjing, Jiangsu, China) and finally normalized to the total protein concentrations of cells.

\section{Alizarin red S (ARS) staining and quantification}

After 14 days of culture in PM or OM, the hASCs were applied to detect the matrix mineralization. Following being fixed with 95\% ethanol for $30 \mathrm{~min}$, cells were soaked in 1\% ARS staining solution (pH 4.2; SigmaAldrich, St. Louis, MO, USA) for $20 \mathrm{~min}$ at room temperature. To assess the degree of mineralization, stained areas of each well were separately dissolved in $100 \mathrm{mM}$ cetylpyridinium chloride (Sigma-Aldrich) for 1 $\mathrm{h}$ and the absorbances were detected at $562 \mathrm{~nm}$. Finally, the relative ARS intensity was normalized to the total protein concentrations of cells.
RNA extraction, reverse transcription, and quantitative real-time polymerase chain reaction (qRT-PCR)

After 3, 7, and 14 days of culture in PM or OM respectively, total RNA of cells was isolated with TRIzol (Invitrogen, Carlsbad, CA, USA) and synthesized into the first-strand cDNA using a reverse transcription system (Takara, Tokyo, Japan). All the transcripts were quantified using the FastStart universal SYBR green master (ROX) (Roche, Indianapolis, IN, USA) and a 7500 realtime PCR detection system (Applied Biosystems, Foster City, CA, USA). Relative expression levels of mRNA and miRNA were normalized to GAPDH mRNA and $U 6$ snRNA, respectively. The sequences of the primers employed are listed in Additional file 6: Table S2.

\section{Western blotting}

The hASCs were rinsed with ice PBS three times and immersed in RIPA buffer (HuaxingBio, Beijing, China) mixed with protease inhibitor cocktail (HuaxingBio). The pierce BCA protein assay kit (Thermo Fisher Scientific) was used to determine the protein concentration. A 25- $\mu$ g sample of protein was added and separated by $10 \%$ sodium dodecyl sulfate-polyacrylamide gel electrophoresis, then followed by transfer to the polyvinylidene difluoride membranes (Millipore, Bedford, MA, USA). Strips on the membranes were blocked with 5\% nonfat dry milk (BioRuler, Danbury, CT, USA) for $1 \mathrm{~h}$ at room temperature, incubated overnight at $4{ }^{\circ} \mathrm{C}$ with primary antibodies at a dilution of 1:1000, and then for $1 \mathrm{~h}$ at room temperature with goat anti-rabbit secondary antibodies labeled with horseradish peroxidase (ZSGB-BIO, Beijing, China; ZB-2301) at a dilution of 1:10,000. The primary antibodies used were as follows: anti-GAPDH (ZSGBBIO; TA-08), anti-NOTCH1 (Cell Signaling Technology, Beverly, MA, USA; 3608S), anti-HES1(Abcam, Cambridge, UK; ab108937), anti-LSD1 (Cell Signaling Technology; 2139S), anti-BMP2 (Abcam; ab14933), anti-SMAD4 (Abcam; ab40759), anti-phosphorylated SMAD1/5 (antip-SMAD1/5; Cell Signaling Technology; 9516S), and antiRUNX2 (Cell Signaling Technology; 12556). Relative band intensities were measured with the ImageJ software.

\section{Dual-luciferase reporter assay}

The 3' untranslated region (3' UTR) alignments of the target regions in NOTCH1 were predicted by TargetScan and RNA22. Reporter vectors were constructed based on the previous method [32]. The 3' UTR sequences of NOTCH1, which contained the possible binding sites of miR-137, were PCR amplified and then inserted into pEZX-MT06 vectors (GeneCopoeia, Rockville, MD, USA) to create NOTCH1-WT (wild-type NOTCH1) luciferase reporter plasmids. Mutated forms were generated by site-directed mutagenesis (GeneCopoeia) and named NOTCH1-MT (mutant-type NOTCH1) luciferase 
reporter plasmids. For luciferase assay, the hASCs were planted on 24-well culture plates with a density of $5 \times$ $10^{4} /$ well and co-transfected with $1 \mu \mathrm{g}$ NOTCH1-WT or NOTCH1-MT plasmids, $100 \mathrm{nM}$ NC or miR-137 mimics, and lipofectamine 3000 (Invitrogen). The luciferase activities were examined by a dual-luciferase reporter assay system (Promega, Madison, WI, USA) $48 \mathrm{~h}$ later, and standardized to renilla luciferase activity for each transfected well.

\section{Heterotopic osteogenesis examinations in vivo}

After the transfection with NC, miR-137 and anti-miR137 lentiviruses, hASCs of the third passage were maintained in PM for 1 week, collected, and incubated with auto-setting calcium phosphate cement (ACPC; Rebone, Shanghai, China) for $1 \mathrm{~h}$ at $37^{\circ} \mathrm{C}$. Then the hASC-ACPC mixtures were transplanted subcutaneously to the dorsal regions of nude mice ( $n=6$ per group) for the analyses of heterotopic bone formation in vivo 8 weeks later. After being collected and fixed in $4 \%$ paraformaldehyde, the samples were photographed with soft X-ray. The radiograph was obtained by applying a Senograph 2000D molybdenum-rhodium twin target X-ray apparatus (GE, Fairfield, CT, USA). The radiation distance is $200 \mathrm{~mm}$ and the radiographing conditions were $22.0 \mathrm{kV}, 35.0 \mathrm{~mA}$. For histological evaluation, the specimens were decalcified in $10 \%$ ethylene diamine tetraacetic acid solution (pH 7.4) for 14 days, embedded into paraffin, then sliced into $5 \mu \mathrm{m}$-thick sections before the subsequent hematoxylin and eosin (HE) staining and Masson trichrome staining. The rabbit anti-OCN primary antibodies diluted to 1:100 (Servicebio, Wuhan, Hubei, China; GB11233) were used for immunohistochemical (IHC) staining.

\section{Statistical analysis}

Data and statistical analyses were conducted with SPSS Statistics 20.0 software (IBM, Armonk, NY, USA). All data were shown as mean \pm standard deviation $(\mathrm{SD})$ of three individual experiments. For the comparison of two independent or multiple groups, Mann-Whitney $U$ test or Kruskal-Wallis test were applied, respectively. A twotailed test with $p$ value $<0.05$ was indicated as statistically significant.

\section{Results}

MiR-137 reversely regulates $\mathrm{hASC}$ differentiation along osteoblastic lineage in vitro

Our previous study displayed an overall downward expression trend of miR-137 in hASCs during the osteoblastic induction and identified its negative role in this biological process [32]. Since lacking other studies on the osteogenic function of miR-137, we re-verified the reliability of our previous results. After transfecting
hASCs with lentiviruses of NC, miR-137 overexpression, and miR-137 knockdown (Additional file 1: Fig. S1a), we evaluated the transfection rate was over $90 \%$ by computing the percentage of GFP-tagged cells (Additional file 1: Fig. S1b). Meanwhile, the transfection effects were quantitatively determined on 3 days, 7 days, and 14 days by qRT-PCR analysis (Additional file 1: Fig. S1c).

ALP staining and activity assays displayed that miR137 overexpression reduced ALP activity of hASCs under proliferation condition or osteogenic induction, but miR-137 knockdown reversed the effects observed with miR-137 overexpression (Additional file 2: Fig. S2a, b). ARS staining and quantification were applied to test the calcium deposits of extracellular matrix. More mineralized nodules were presented in miR-137 knockdown group while less in miR-137 overexpression group when both were compared with $\mathrm{NC}$ group (Additional file 2: Fig. S2c, d). Besides, the characteristic genes expressed in different stages of osteogenesis, including RUNX2, $A L P$, and $O C N$, were examined by qRT-PCR and presented significant decreases in miR-137 overexpression group but dramatically increased in miR-137 knockdown group (Additional file 2: Fig. S2e). According to these data, we substantiated that miR-137 inhibits in vitro osteoblastic activity of hASCs.

\section{MiR-137 reversely regulates hASC differentiation along osteoblastic lineage in vivo}

In order to validate in vivo osteogenic effects of miR137, hASCs were transfected with NC, miR-137, and anti-miR-137 lentiviruses and separately mixed with $\mathrm{ACPC}$, and then the compounds were subcutaneously implanted into the dorsum of nude mice (Additional file 3: Fig. S3a). After 8 weeks, the total volume and mean density of the harvested samples were assessed and manifested an apparent enhancement in miR-137 knockdown group but remarkable reduction in miR-137 overexpression group (Additional file 3: Fig. S3b, c).

Histological analyses of bone formation were performed by staining of HE, Masson trichrome, and IHC staining for OCN. HE staining showed more new bone formation in miR-137 knockdown group when comparing with NC group, which displayed only a very small amount of osteoid, but we could hardly observe any new bone or osteoid in miR-137 overexpression group. Similarly, thicker and more compact blue-green-stained collagen fiber bundles were detected in miR-137 knockdown group than in another two groups, but overexpression of miR-137 led to the thinnest collagen deposition. Moreover, we found that dark-brown stained OCN granules were the most widespread in the cells of miR-137 knockdown group, fewer in NC group, and none could be discerned in miR-137 overexpression group (Additional file 3: Fig. S3d). Consequently, in accordance with 
the results of in vitro experiments, miR-137 subdues the osteoblastic activity of hASCs in vivo.

\section{MiR-137 regulates NOTCH1-HES1 pathway by directly targeting $\mathrm{NOTCH} 1$}

To ascertain the influences of miR-137 on NOTCH1 pathway, we first examined the expression of $\mathrm{NOTCH1}$ and its downstream signal HES1 with miR-137 overexpression or knockdown. When compared with NC group, the mRNA and protein levels of NOTCH1 showed obvious increase in miR-137 knockdown group while marked reduction in miR-137 overexpression group. Contrary to NOTCH1, the expression tendency of $H E S 1$ accorded with the changes of miR-137 (Fig. 1a-c). These findings suggested that NOTCH1 is negatively regulated while HES1 is positively regulated by miR-137.

To further identify whether miR-137 could directly bind to NOTCH1 in hASCs as it does in other cell lines [4044], dual-luciferase reporter assays were carried out. The presumed targeting sites of miR-137 in the 3' UTR of NOTCH1 were forecasted by two prediction softwares (TargetScan and RNA22). Then the luciferase reporter vectors carrying the 3' UTR of NOTCH1-WT or NOTCH1-MT were constructed (Fig. 1d, e) and the

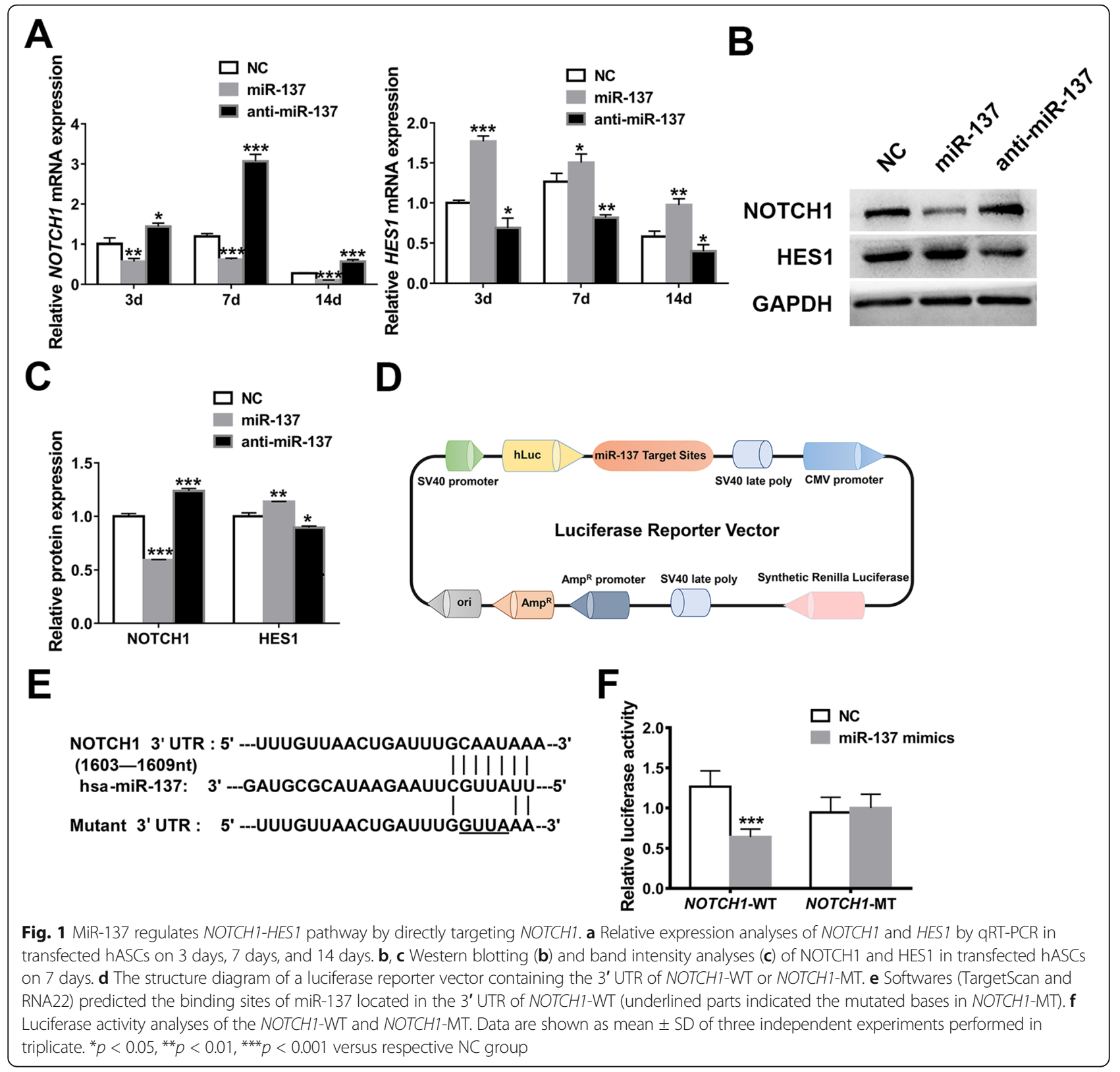


relative luciferase activities were detected. MiR-137 mimics significantly repressed the luciferase activity in NOTCH1-WT group while they had no significant influences in NOTCH1-MT group when both groups were compared with their respective NC group (Fig. 1f). These results validated that miR-137 directly binds to the 3' UTR of NOTCH1 and induces the expression of HES1 in hASCs.
NOTCH1 inhibitor reverses the effects of miR-137 knockdown on osteogenesis and downstream genes expression

To confirm whether miR-137 regulated osteogenesis through NOTCH1, we employed NOTCH1 inhibitor (tangeretin) in hASCs transfected with miR-137 knockdown lentiviruses. In vitro osteogenic stainings and quantification manifested that when compared with NC

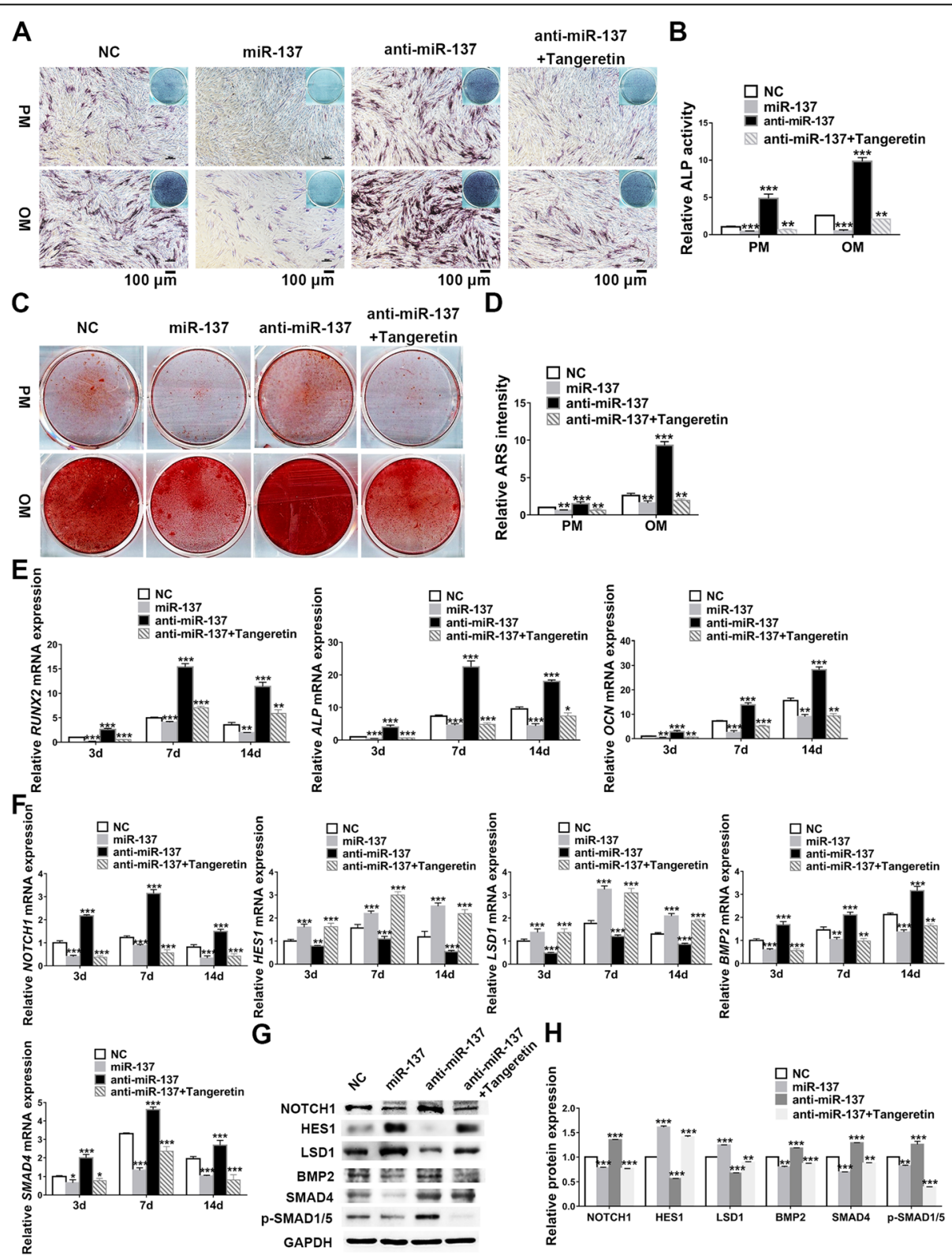

Fig. 2 NOTCH1 inhibitor (tangeretin) reverses the effects of miR-137 knockdown on osteogenesis and downstream genes expression. a, b ALP staining (a) and quantification (b) of transfected hASCs after a 7-day culture in PM or OM (scale bar $=100 \mu \mathrm{m})$. c $\mathbf{d}$ d ARS staining (c) and quantification (d) of transfected hASCs after a 14-day culture in PM or OM. e Relative expression analyses of RUNX2, ALP, and OCN by qRT-PCR in transfected hASCs on 3 days, 7 days, and 14 days. $\mathbf{f}$ Relative expression analyses of NOTCH1, HES1, LSD1, BMP2, and SMAD4 by qRT-PCR in transfected hASCs on 3 days, 7 days, and 14 days. g, h Western blotting (g) and band intensity analyses (h) of NOTCH1, HES1, LSD1, BMP2, SMAD4, and p-SMAD1/5 in transfected hASCs on 7 days. Data are shown as mean \pm SD of three independent experiments performed in triplicate. ${ }^{*} p<0.05,{ }^{* *} p<0.01,{ }^{* * *} p<0.001$ versus respective NC group 
group, miR-137 knockdown evidently strengthened ALP activity and extracellular matrix calcification, whereas the promoted osteogenic ability caused by miR-137 knockdown were completely reversed by tangeretin treatment, and miR-137 overexpression group displayed impaired osteogenic differentiation potential as previously mentioned (Fig. 2a-d). In accord with the results of osteogenic stainings, qRT-PCR detection showed that the expression of $R U N X 2, A L P$, and $O C N$ were enhanced in miR-137 knockdown group while reduced in miR-137 overexpression group, but the addition of tangeretin abrogated the induction of these genes by miR137 knockdown and even suppressed their expression (Fig. 2e).

To further authenticate whether NOTCH1 mediated the mechanisms of the osteogenic regulation by miR-
A

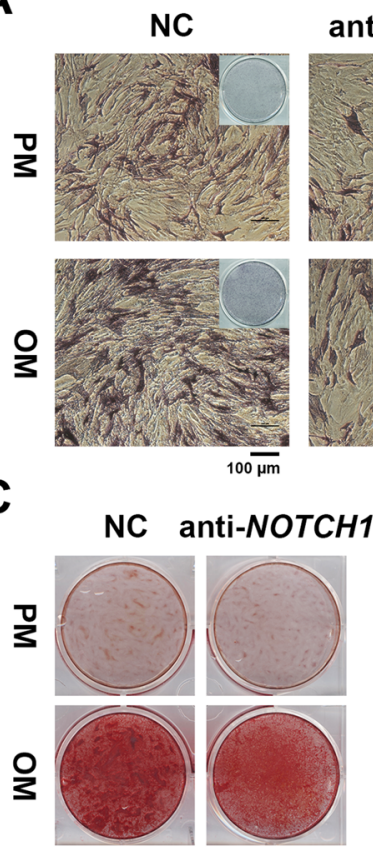

E
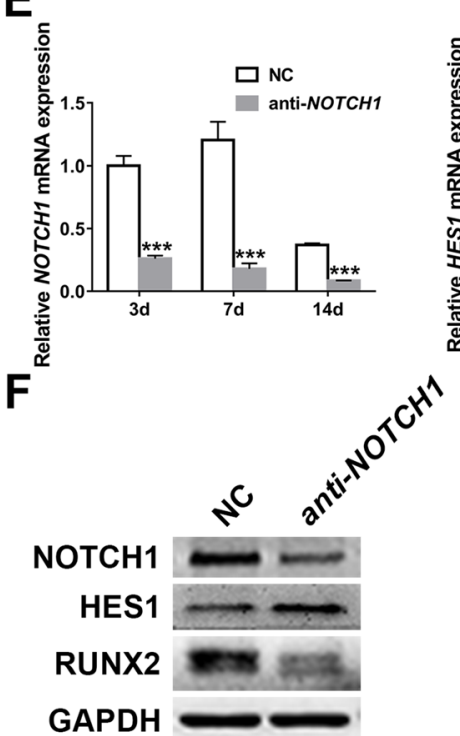

B

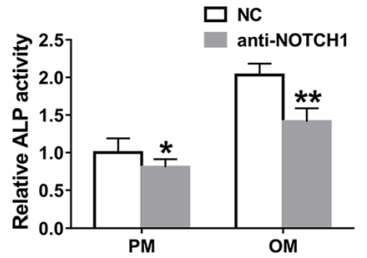

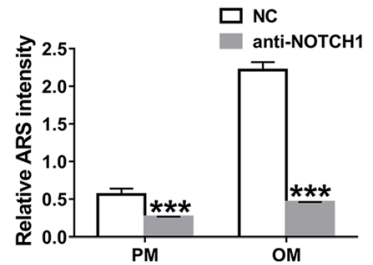
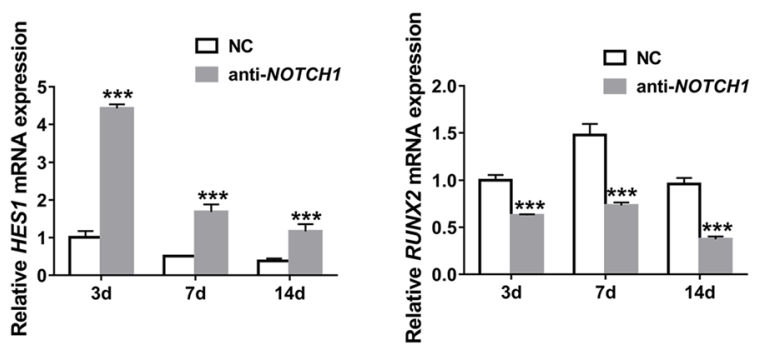

G

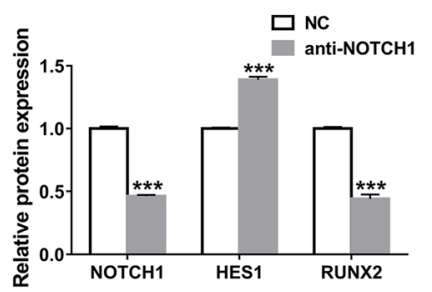

Fig. 3 NOTCH1 knockdown impairs osteogenesis by inducing HES1. a, b ALP staining (a) and quantification (b) of transfected hASCs after a 7-day culture in PM or OM (scale bar = $100 \mu \mathrm{m})$. c, d ARS staining (c) and quantification (d) of transfected hASCs after a 14-day culture in PM or OM. e Relative expression analyses of NOTCH1, HES1, and RUNX2 by qRT-PCR in transfected hASCs on 3 days, 7 days, and 14 days. f, $\mathbf{g}$ Western blotting (f) and band intensity analyses ( $\mathbf{g}$ ) of NOTCH1, HES1, and RUNX2 in transfected hASCs on 7 days. Data are shown as mean \pm SD of three independent experiments performed in triplicate. ${ }^{*} p<0.05,{ }^{* *} p<0.01,{ }^{* * *} p<0.001$ versus respective NC group 
137, we examined the expression of miR-137 downstream signals in the above four groups. Both in miR137 overexpression and tangeretin-treated groups, NOTCH1 was significantly repressed, though miR-137 knockdown induced its expression. The mRNA and protein expression showed that HES1 and LSD1 changed synchronously with the alterations of miR-137, but tangeretin-treated group inverted the downregulation of these two genes by miR-137 knockdown when compared with NC group. In addition, the mRNA or protein expression levels of BMP2, SMAD4, and p-SMAD1/5 showed that these genes were induced by miR-137 knockdown while inhibited by miR-137 overexpression. Similarly, tangeretin inhibited the activation of BMP2-
SMADs pathway initiated by miR-137 knockdown (Fig. $2 \mathrm{f}-\mathrm{h})$. Combined with the above results, we affirmed that NOTCH1 inhibitor reverses the osteogenic modulation effects of miR-137 knockdown.

\section{NOTCH1 knockdown impairs osteogenesis by inducing HES1}

To determine the influences of NOTCH1 knockdown on the osteoblastic potential of hASCs, we applied ALP and ARS stainings combined with quantitative analysis and found that NOTCH1 knockdown attenuated ALP activity and extracellular mineralization (Fig. 3a-d). As the downstream molecules of NOTCH1, HES1, and RUNX2 were further detected at
A

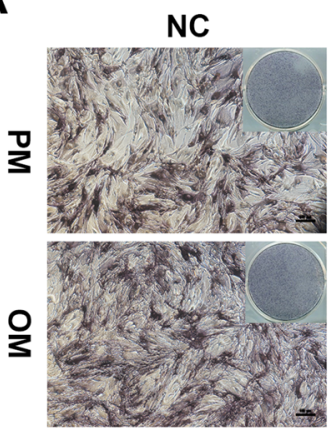

$100 \mu \bar{m}$

C

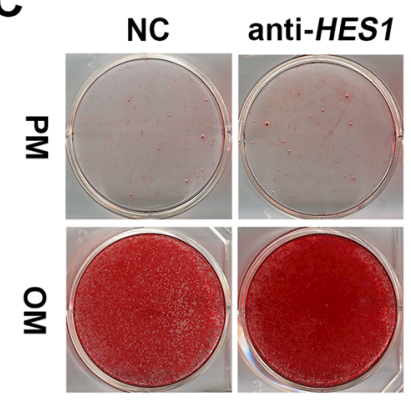

D
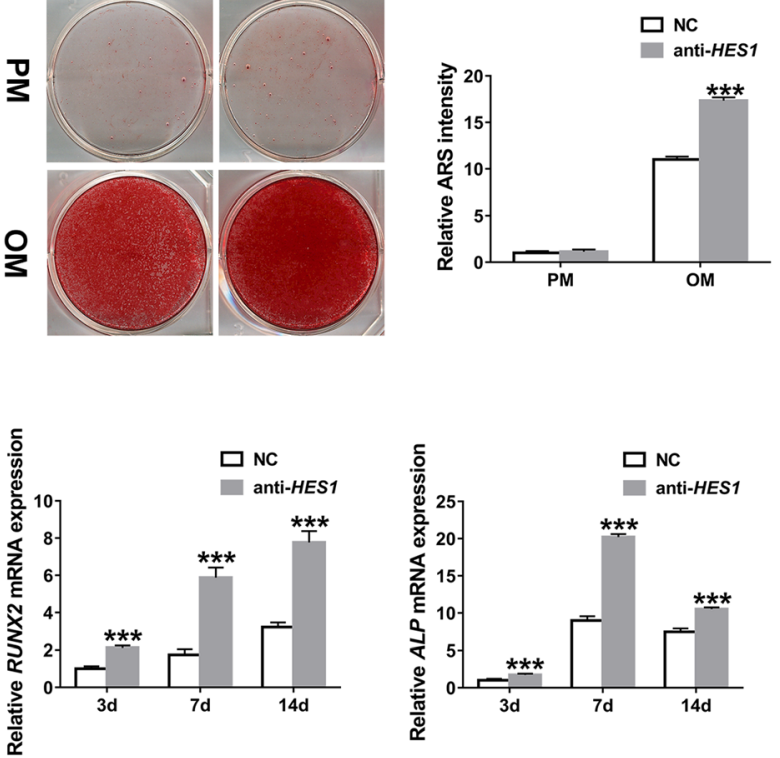

anti-HES1

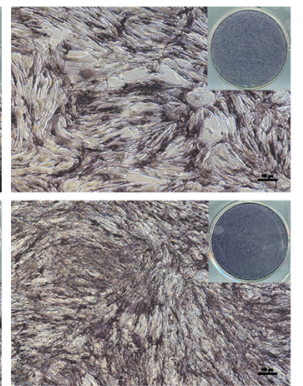

$100 \mu \bar{m}$

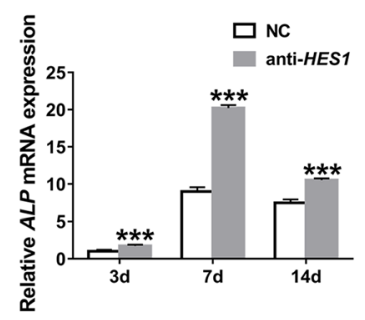

B

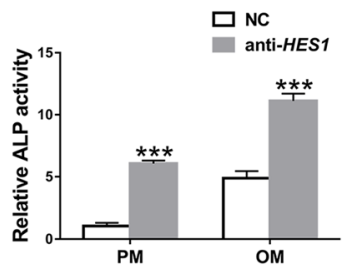

E
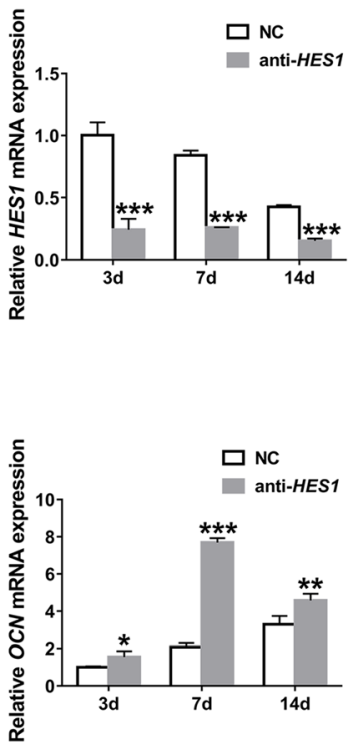

Fig. 4 HES1 knockdown promotes osteogenesis of hASCs in vitro. a, b ALP staining (a) and quantification (b) of transfected hASCS after a 7-day culture in PM or OM (scale bar $=100 \mu \mathrm{m})$. $\mathbf{c}$, d ARS staining (c) and quantification (d) of transfected hASCs after a 14-day culture in PM or OM. $\mathbf{e}$ Relative expression analyses of HES1, RUNX2, ALP, and OCN by qRT-PCR in transfected hASCs on 3 days, 7 days, and 14 days. Data are shown as mean \pm SD of three independent experiments performed in triplicate. ${ }^{*} p<0.05,{ }^{* *} p<0.01,{ }^{* * *} p<0.001$ versus respective NC group 
mRNA and protein levels in hASCs transfected with NOTCH1 knockdown lentiviruses. Coincident with the impacts of miR-137 on NOTCH1-HES1 pathway, NOTCH1 knockdown induced the expression of HES1 while repressed RUNX2 (Fig. $3 \mathrm{e}-\mathrm{g}$ ). Our results indicated that NOTCH1 knockdown impedes the osteogenic potential of hASCs by the stimulation of HES1, corroborating the former conclusions that miR-137 inhibits osteogenesis by the downregulation of NOTCH1 and upregulation of HES1.

Then we checked the impacts of HES1 on the osteogenic differentiation of hASCs. After knocking down HES1, we examined the osteogenic ability by the application of osteogenic stainings and quantification as well as qRT-PCR detection of RUNX2, ALP, and $O C N$. All these in vitro osteogenic tests showed promoted osteogenic potential of hASCs with HES1 knockdown (Fig. 4). Therefore, we demonstrated that HES1 plays a negative role in the osteoblastic differentiation of hASCs and intervenes in NOTCH1induced osteogenesis.

\section{NOTCH1 knockdown induces LSD1 while inhibits BMP2- SMADs pathway}

Since our previous study has demonstrated that miR-137 upregulated $L S D 1$ while downregulated $B M P 2$ and SMAD4 in hASCs [32], we further investigated the influences of NOTCH1 knockdown on LSD1 and BMP2-SMADs pathway. As predicted, the expression of $L S D 1$ significantly increased after knocking down NOTCH1 while BMP2, SMAD4, and p-SMAD1/5 decreased apparently (Fig. 5). Therefore, we deduced that the osteogenic inhibition of NOTCH1 knockdown is also dependent on the activation of $L S D 1$ and suppression of BMP2-SMADs pathway.

\section{LSD1 knockdown regulates NOTCH1-HES1 pathway}

As above, we have affirmed that $\mathrm{NOTCH1}$ acted as a negative regulator in LSD1 expression. But considering the

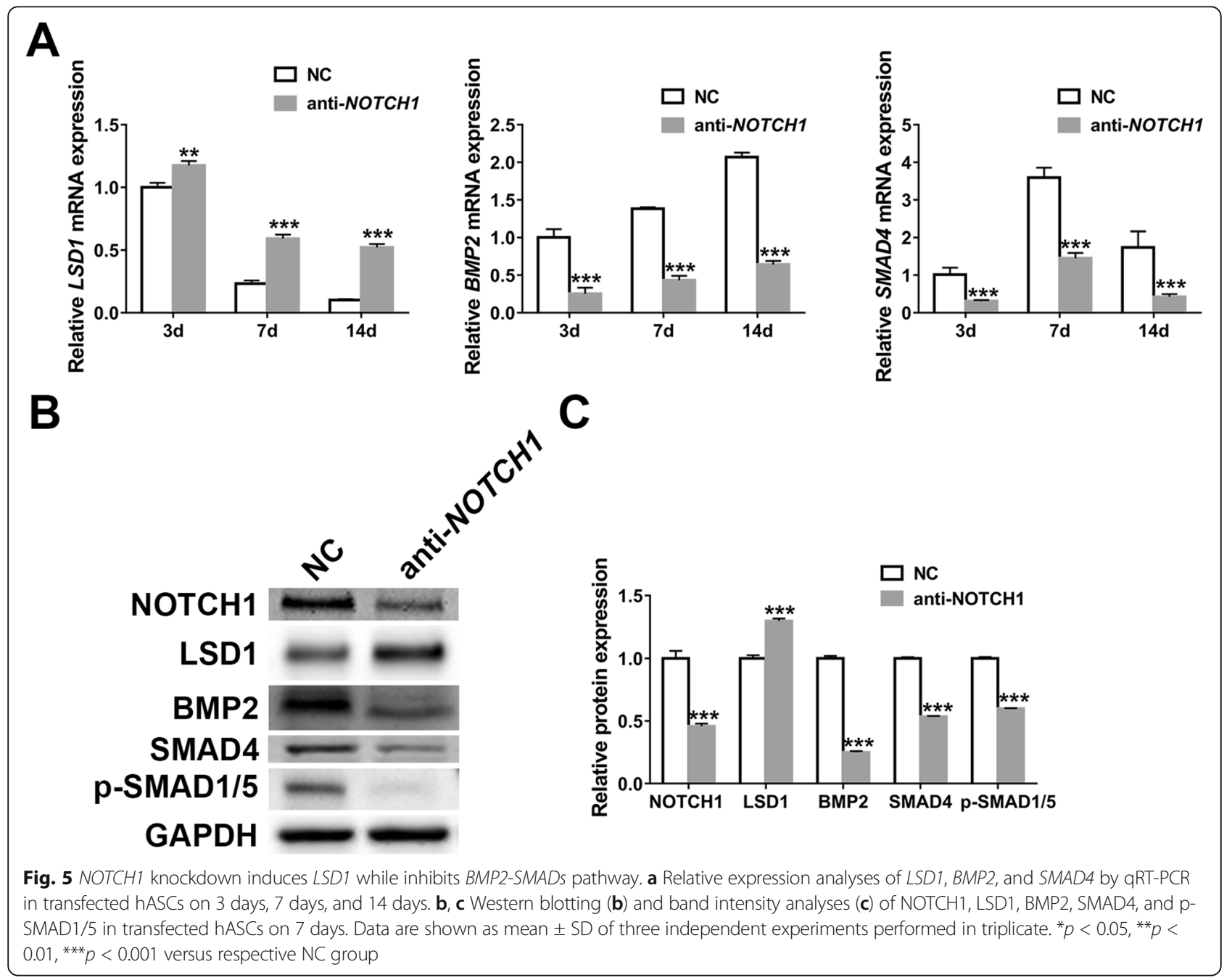




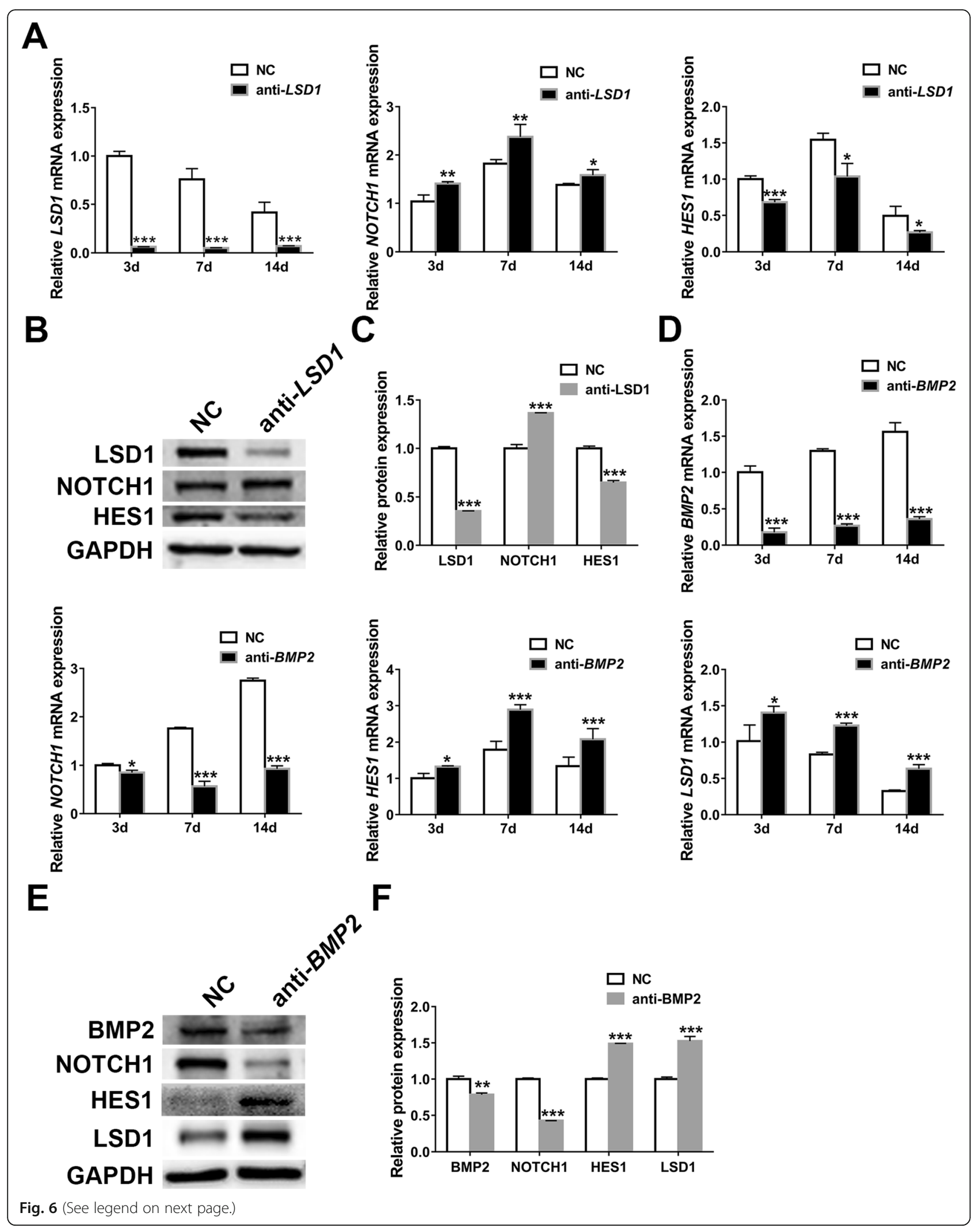


(See figure on previous page.)

Fig. 6 LSD1 or BMP2 knockdown influences NOTCH1/LSD1/BMP2 signaling network. a Relative expression analyses of LSD1, NOTCH1, and HES1 by qRT-PCR in transfected hASCs on 3 days, 7 days, and 14 days. b, c Western blotting (b) and band intensity analyses (c) of LSD1, NOTCH1, and HES1 in transfected hASCs on 7 days. d Relative expression analyses of BMP2, NOTCH1, HES1, and LSD1 by qRT-PCR in transfected hASCs on 3 days, 7 days, and 14 days. e, f Western blotting (e) and band intensity analyses (f) of BMP2, NOTCH1, HES1, and LSD1 in transfected hASCs on 7 days. Data are shown as mean \pm SD of three independent experiments performed in triplicate. ${ }^{*} p<0.05,{ }^{* *} p<0.01,{ }^{* * *} p<0.001$ versus respective NC group

complex interplay of signaling molecules, we tried to clarify whether LSD1 had feedback effects on NOTCH1-HES1 pathway. Notably, we found that LSD1 knockdown led to a higher level of NOTCH1 while lowered the expression of HES1 when compared with $\mathrm{NC}$ group (Fig. 6a-c), thus prompting a reciprocal negative relationship between NOTCH1 and LSD1.

\section{BMP2 knockdown inhibits HOTCH1 while induces HES1 and $L S D 1$}

To gain further insights into the relationships between NOTCH1, LSD1, and BMP2 signals, we then examined the expression of NOTCH1, HES1, and LSD1 at mRNA and protein levels after knocking down $B M P 2$. Importantly, the expression of NOTCH1 dramatically decreased while $H E S 1$ and $L S D 1$ increased with $B M P 2$ knockdown (Fig. 6d-f). Combining with the above results that NOTCH1 knockdown inhibited BMP2-SMADs pathway, we verified a positive feedback loop between NOTCH1 and BMP2. Furthermore, our previous study revealed that silencing of $L S D 1$ promoted the osteoblastic potential of hASCs by stimulating BMP2-SMAD4 signaling pathway [32]. Here again, we observed upward tendencies in the expression of BMP2, SMAD4, RUNX2, and $A L P$ with $L S D 1$ knockdown (Additional file 4: Fig. S4). Therefore, a negative interplay between $L S D 1$ and $B M P 2$ was also confirmed by us.

\section{Discussion}

Dysfunction of miR-137 has been implicated in several cancer types such as glioblastoma [19], melanoma [20], multiple myeloma [21], non-small cell lung cancer [22], and endometrial cancer [23]. In addition, miR-137 has been found to play an essential role in neural development and maturation, with several studies displaying an association with cell proliferation and neurogenic differentiation [24-31]. However, little is known concerning its functions and regulatory mechanisms on the osteoblastic differentiation, especially in mesenchymal stem cells. Silencing of miR-137-3p is found to facilitate the osteogenesis of bone marrow-derived mesenchymal stem cells by targeting RUNX2 [50]. Previously, we demonstrated that silencing of miR-137 promoted the osteoblastic activity in hASCs and revealed its modulation on the signaling network of $L S D 1 / B M P 2 / S M A D 4$ as part of the mechanisms [32]. Interestingly, our former research

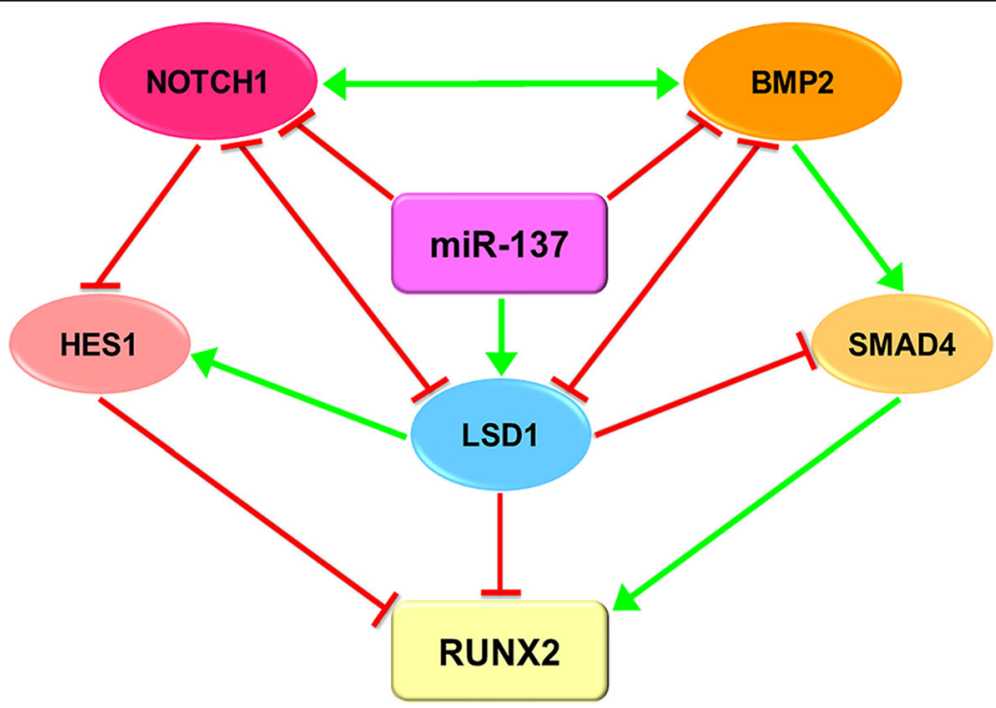

Fig. 7 A diagrammatic view of NOTCH1/LSD1/BMP2 signaling network in miR-137-mediated differentiation of hASCs towards osteoblastic lineage. In this complex network model, miR-137 modulates NOTCH1-HES1, LSD1, and BMP2-SMADs pathways simultaneously. Depending on the positive feedback loop between NOTCH1 and BMP2, as well as the negative reciprocal relationship between LSD1 and NOTCH1 or BMP2, the impacts of miR-137 on the above three signaling pathways are strengthened and the NOTCH1/LSD1/BMP2 network regulates the osteogenesis of hASCS coordinately (red lines represent downregulation and green lines represent upregulation) 
confirmed a positive role of miR-137 in LSD1 expression, which was contrary to several studies reporting that miR-137 directly binds to $L S D 1$ [23, 26, 51, 52]. The contradicted outcomes might be associated with the various biological features of different cell types, and we deduced that there probably exist intermediary regulators working between miR-137 and LSD1 during the osteogenesis of hASCs. Notably, this study identified that NOTCH1 was a direct target of miR-137 in hASCs, and NOTCH1-HES1 pathway was engaged in the crosstalk between $L S D 1$ and BMP2-SMADs pathway. In this way, NOTCH1 signal mediated the control of miR-137 on LSD1/BMP2/SMAD4 network. Moreover, the interrelations of the above signals were validated comprehensively and a NOTCH1/LSD1/BMP2 co-regulatory network was established, further elucidating the epigenetic mechanisms of miR-137 during the process of hASCs differentiating into osteoblastic lineage.

After reconfirming the inhibitory impacts of miR-137 on the osteoblastic activity of hASCs both in vitro and in vivo, we demonstrated that miR-137 negatively regulated the expression of $\mathrm{NOTCH1}$ while positively regulated HES1. NOTCH signaling pathway influences tumorigenesis as well as embryonic development [53] because of its crucial role in cell fate determination, proliferation, differentiation, and apoptosis [54]. Though it is still debatable whether NOTCH signal serves as a positive or negative regulator in osteogenesis [55], our data displayed impaired osteogenic capacity of hASCs after knocking down NOTCH1. Most noteworthy, NOTCH1 was validated as a direct target gene of miR-137 in hASCs, the same as in other cell lines [40-44]. HES1 is known as a potential downstream gene of NOTCH1 in many studies, but it is not affected in NOTCH1 knockout mice while the expression of HES5 and HES-related repressor protein $(H E R P) 1,-2$, and -3 are greatly diminished [56-58]. As a transcriptional regulator in the NOTCH signaling pathway, recombination signal binding protein (RBPJ) gene disruption in homozygous mice exhibits reduced HES5 expression, but not for HES1 [56]. Given the various effects on OCN, osteopontin, and RUNX2 [37, 38], HES1 influences the osteogenesis inconsistently depending on the different cellular environments. This study exhibited enhanced osteogenic differentiation of hASCs with HES1 knockdown. Moreover, NOTCH1 knockdown upregulated HES1, indicating that NOTCH1 acts as a negative regulator in the expression of HES1. To further identify whether NOTCH1 mediated the osteogenic differentiation of hASCs modulated by miR-137, we applied NOTCH1 inhibitor (tangeretin) in hASCs transfected with miR-137 knockdown lentiviruses and found that tangeretin reversed the impacts of miR-137 knockdown on osteogenesis and downstream genes expression, thus verifying the vital role of NOTCH1 in the osteogenic regulation of miR-137. Collectively, we brought insight into how the NOTCH1-HES1 pathway was influenced and involved in the osteogenic modulation of miR-137.

LSD1 has been linked to the repression of NOTCH1 pathway in various cell types [45, 59-63], though one study states that it functions as a corepressor when associated with RBPJ-repressor complex and as a NOTCH1 coactivator upon $\mathrm{NOTCH}$ activation [64]. Nevertheless, few studies have reported the interplay between NOTCH1 and LSD1 during the osteogenesis of hASCs and whether this interaction contributes to the osteogenic control of miR-137 is still unknown. Coincident with the influences of miR-137 on NOTCH1 and LSD1, we uncovered a negative interaction between NOTCH1 and $L S D 1$ with separate knockdown of them. More intriguingly, despite inducing the expression of NOTCH1, LSD1 knockdown repressed HES1. Thus, the opposite expression trends of NOTCH1 and HES1 caused by LSD1 knockdown might reinforce the downregulation of HES1 by NOTCH1 alone. These results authenticated the crosstalk between NOTCH1-HES1 pathway and LSD1, through which miR-137 regulates the osteogenic differentiation of hASCs.

$B M P$ signal is a canonical pathway in skeleton and $B M P 2-S M A D 4$ pathway has been shown to participate in the osteogenic regulation of miR-137 by us [32]. After knocking down NOTCH1 or BMP2 individually, we observed suppressed expression of NOTCH1, BMP2, and $S M A D s$ simultaneously, indicating a positive interrelationship between NOTCH1 and BMP2-SMADs pathway. Besides, increased HES1 expression with BMP2 knockdown proved the negative effects of NOTCH1 on HES1 from another aspect. Considering our previous results that LSD1 knockdown activated the BMP2-SMAD4 pathway [32], in turn, we investigated the impacts of $B M P 2$ on $L S D 1$. As expected, $L S D 1$ was significantly upregulated with $B M P 2$ knockdown. Thus, these findings suggested a negative feedback loop between $L S D 1$ and BMP2-SMADs pathway.

In conclusion, our results revealed that depending on the reciprocal negative regulation between $\mathrm{NOTCH} 1$ and $L S D 1, L S D 1$ and $B M P 2$, as well as the synergistic function between NOTCH1 and BMP2, miR-137 negatively regulates osteogenesis of hASCs through the NOTCH1/ LSD1/BMP2 co-regulatory signaling network (Fig. 7).

\section{Conclusions}

In summary, our study provided a relatively comprehensive rationale for the negative modulation of miR-137 during the hASC differentiation towards osteoblastic lineage and established a co-regulatory network of NOTCH1/LSD1/BMP2 to elucidate the underlying 
mechanisms, which is of substantial importance for potential targeted therapy of bone-related diseases.

\section{Abbreviations}

3' UTR: 3' untranslated region; ACPC: Auto-setting calcium phosphate cement; ALP: Alkaline phosphatase; ARS: Alizarin red S; BMP: Bone morphogenetic protein; GFP: Green fluorescent protein; hASCs: Human adipose-derived stem cells; HE: Hematoxylin and eosin; HES1: Hairy and enhancer of split 1; IHC: Immunohistochemical; LSD1: Lysine-specific histone demethylase 1; miRNA: MicroRNA; OCN: Osteocalcin; OM: Osteogenic medium; PM: Proliferation medium; qRT-PCR: Quantitative real-time polymerase chain reaction; RUNX2: Runt-related transcription factor 2 SMAD4: Mothers against decapentaplegic homolog 4; p-SMAD1/ 5: Phosphorylated SMAD1/5

\section{Supplementary Information}

The online version contains supplementary material available at https://doi. org/10.1186/s13287-021-02495-3.

\section{Additional file 1: Figure S1. Efficiency determination of lentiviral} transfection. a The structure diagram of packaged lentiviruses. b Microscopic images of transfected hASCs with GFP-tagged lentiviruses under the ordinary (left panel) and fluorescent light (right panel). Scale bar $=100 \mu \mathrm{m}$. c Relative expression analysis of miR-137 by qRT-PCR in transfected hASCs on $3 \mathrm{~d}, 7 \mathrm{~d}$ and $14 \mathrm{~d}$. Data are shown as mean \pm SD of three independent experiments performed in triplicate. ${ }^{*} p<0.05,{ }^{* *} p$ $<0.01,{ }^{* * *} p<0.001$ versus respective NC group.

Additional file 2: Figure S2. MiR-137 reversely regulates hASC differentiation along osteoblastic lineage in vitro. a, b ALP staining (a) and quantification (b) of transfected hASCs after a 7-day culture in PM or OM (scale bar $=100 \mu \mathrm{m}) . c$, d ARS staining (c) and quantification (d) of transfected hASCs after a 14-day culture in PM or OM. e Relative expression analyses of RUNX2, ALP and OCN by qRT-PCR in transfected hASCs on $3 \mathrm{~d}, 7 \mathrm{~d}$ and $14 \mathrm{~d}$. Data are shown as mean \pm SD of three independent experiments performed in triplicate. ${ }^{*} p<0.05,{ }^{* *} p<0.01,{ }^{* *} p<0.001$ versus respective NC group.

Additional file 3: Figure S3. MiR-137 reversely regulates hASC differentiation along osteoblastic lineage in vivo. a Schema of the experimental design for in vivo study. $n=6$ per group. b Representative soft X-ray photographs of the specimens which were subcutaneously harvested from the dorsal pockets of nude mice 8 weeks later. c Mean density analyses by the application of ImageJ software. Data are shown as mean \pm SD of six independent experiments. ${ }^{*} p<0.05,{ }^{* *} p<0.01,{ }^{* * *} p<0.001$ versus NC group. d Heterotopic bone formation was evaluated by histological stainings: $\mathrm{HE}$, Masson trichrome staining (scale bar $=50 \mu \mathrm{m}$ ), and $\mathrm{IHC}$ staining of OCN (scale bar $=20 \mu \mathrm{m}$ ). Typical dark brown particles indicating OCN depositions in hASCs were marked with black arrows.

Additional file 4: Figure S4. LSD1 knockdown activates BMP2-SMAD4 pathway and osteogenesis-associated genes expression. a Relative expression analyses of BMP2, SMAD4, RUNX2 and ALP by qRT-PCR in transfected hASCS on $3 \mathrm{~d}, 7 \mathrm{~d}$ and $14 \mathrm{~d}$. b, c Western blotting (b) and band intensity analyses (c) of LSD1, BMP2, SMAD4 and RUNX2 in transfected hASCs on 7 d. Data are shown as mean \pm SD of three independent experiments performed in triplicate. ${ }^{*} \mathrm{p}<0.05,{ }^{* *} p<0.01,{ }^{* * *} p<0.001$ versus respective NC group.

Additional file 5: Table S1. Sequences for lentiviral vectors.

Additional file 6: Table S2. Sequences of the primers for qRT-PCR.

Additional file 7: Table S3. Key resources table.

\section{Acknowledgements}

Not applicable.

\section{Authors' contributions}

CF conceived the project, provided financial supports, and wrote the manuscript. XM performed the main experiments, analyzed data and prepared figures. YW, LL, YZ, and $H L$ helped with the animal work. $Y L$ assisted with the data analysis and offered insightful ideas.

\section{Funding}

This study was financially granted by grants from the National Natural Science Foundation of China (No. 81700937 and No. 81930026), and Beijing Natural Science Foundation (No. L182006).

Availability of data and materials

The datasets used and analyzed during the current study are available from the corresponding author on reasonable request.

\section{Declarations}

Ethics approval and consent to participate

This study was approved by the Institutional Animal Care and Use Committee of the Peking University Health Science Center (Approval No. LA2019019).

\section{Consent for publication}

Not applicable.

\section{Competing interests}

The authors declare that they have no competing interests.

\section{Author details}

${ }^{1}$ Department of General Dentistry II, Peking University School and Hospital of Stomatology, Beijing, China. ${ }^{2}$ National Center of Stomatology, Beijing, China. ${ }^{3}$ National Clinical Research Center for Oral Diseases, Beijing, China. ${ }^{4}$ National Engineering Laboratory for Digital and Material Technology of Stomatology, Beijing, China. ${ }^{5}$ Research Center of Engineering and Technology for Computerized Dentistry Ministry of Health, Beijing, China. ${ }^{6}$ NMPA Key Laboratory for Dental Materials, Beijing, China. ${ }^{7}$ Department of Prosthodontics, Peking University School and Hospital of Stomatology, Beijing, China. ${ }^{8}$ Department of Prosthodontics, Beijing Stomatological Hospital Capital Medical University, Beijing, China. ${ }^{9}$ Central Laboratory, Peking University School and Hospital of Stomatology, Beijing, China.

Received: 31 August 2020 Accepted: 5 July 2021

Published online: 22 July 2021

\section{References}

1. Deng Y, Zhou H, Zou D, Xie Q, Bi X, Gu P, et al. The role of miR-31-modified adipose tissue-derived stem cells in repairing rat critical-sized calvarial defects. Biomaterials. 2013;34(28):6717-28. https://doi.org/10.1016/j.bioma terials.2013.05.042

2. Hao W, Liu H, Zhou L, Sun Y, Su H, Ni J, et al. MiR-145 regulates osteogenic differentiation of human adipose-derived mesenchymal stem cells through targeting FoxO1. Exp Biol Med (Maywood). 2018;243(4):386-93. https://doi. org/10.1177/1535370217746611.

3. Lin CY, Chang YH, Li KC, Lu CH, Sung LY, Yeh CL, et al. The use of ASCs engineered to express BMP2 or TGF- $\beta 3$ within scaffold constructs to promote calvarial bone repair. Biomaterials. 2013;34(37):9401-12. https://doi. org/10.1016/j.biomaterials.2013.08.051

4. Ullah I, Subbarao RB, Rho GJ. Human mesenchymal stem cells-current trends and future prospective. Biosci Rep. 2015;35(2):e00191. https://doi. org/10.1042/BSR20150025.

5. Zhang WB, Zhong WJ, Wang L. A signal-amplification circuit between miR218 and Wnt/ $\beta$-catenin signal promotes human adipose tissue-derived stem cells osteogenic differentiation. Bone. 2014;58:59-66. https://doi.org/10.101 6/j.bone.2013.09.015

6. Becker M, Potapenko T, Niklaus A, Bieback K, Ho AD, Müller AM. Polycomb protein BMI1 regulates osteogenic differentiation of human adipose tissuederived mesenchymal stem cells downstream of GSK3. Stem Cells Dev. 2016;25(12):922-33. https://doi.org/10.1089/scd.2015.0277.

7. Quarto N, Senarath-Yapa K, Renda A, Longaker MT. TWIST1 silencing enhances in vitro and in vivo osteogenic differentiation of human adiposederived stem cells by triggering activation of BMP-ERK/FGF signaling and TAZ upregulation. Stem Cells. 2015;33(3):833-47. https://doi.org/10.1002/ stem.1907.

8. Vanhatupa S, Ojansivu M, Autio R, Juntunen M, Miettinen S. Bone morphogenetic protein-2 induces donor-dependent osteogenic and adipogenic differentiation in human adipose stem cells. Stem Cells Transl Med. 2015;4(12):1391-402. https://doi.org/10.5966/sctm.2015-0042. 
9. Zhang Z, Ma Y, Guo S, He Y, Bai G, Zhang W. Low-intensity pulsed ultrasound stimulation facilitates in vitro osteogenic differentiation of human adipose-derived stem cells via up-regulation of heat shock protein (HSP)70, HSP90, and bone morphogenetic protein (BMP) signaling pathway. Biosci Rep. 2018;38(3):BSR20180087. https://doi.org/10.1042/BSR20180087.

10. Fan C, Jia L, Zheng Y, Jin C, Liu Y, Liu H, et al. MiR-34a promotes osteogenic differentiation of human adipose-derived stem cells via the RBP2/NOTCH1/ CYCLIN D1 coregulatory network. Stem Cell Reports. 2016;7(2):236-48. https://doi.org/10.1016/j.stemcr.2016.06.010.

11. Lough DM, Chambers C, Germann G, Bueno R, Reichensperger J, Swanson E, et al. Regulation of ADSC osteoinductive potential using Notch pathway inhibition and gene rescue: a potential on/off switch for clinical applications in bone formation and reconstructive efforts. Plast Reconstr Surg. 2016; 138(4):642e-52e.

12. Aimaiti A, Maimaitiyiming A, Boyong X, Aji K, Li C, Cui L. Low-dose strontium stimulates osteogenesis but high-dose doses cause apoptosis in human adipose-derived stem cells via regulation of the ERK1/2 signaling pathway. Stem Cell Res Ther. 2017;8(1):282. https://doi.org/10.1186/s13287017-0726-8.

13. Zhang $M$, Zhang $P$, Liu Y, LV L, Zhang $X$, Liu H, et al. RSPO3-LGR4 regulates osteogenic differentiation of human adipose-derived stem cells via ERK/FGF signalling. Sci Rep. 2017;7(1):42841. https://doi.org/10.1038/srep42841.

14. Ambros V. The functions of animal microRNAs. Nature. 2004;431(7006):3505. https://doi.org/10.1038/nature02871.

15. Chen S, Zheng Y, Zhang S, Jia L, Zhou Y. Promotion effects of miR-375 on the osteogenic differentiation of human adipose-derived mesenchymal stem cells. Stem Cell Reports. 2017;8(3):773-86. https://doi.org/10.1016/j. stemcr.2017.01.028.

16. Kim YJ, Bae SW, Yu SS, Bae YC, Jung JS. MiR-196a regulates proliferation and osteogenic differentiation in mesenchymal stem cells derived from human adipose tissue. J Bone Miner Res. 2009;24(5):816-25. https://doi.org/10.1359/ jbmr.081230.

17. Liao YH, Chang YH, Sung LY, Li KC, Yeh CL, Yen TC, et al. Osteogenic differentiation of adipose-derived stem cells and calvarial defect repair using baculovirus-mediated co-expression of BMP-2 and miR-148b. Biomaterials. 2014;35(18):4901-10. https://doi.org/10.1016/j.biomaterials.2014.02.055.

18. Zeng Y, Qu X, Li H, Huang S, Wang S, Xu Q, et al. MicroRNA-100 regulates osteogenic differentiation of human adipose-derived mesenchymal stem cells by targeting BMPR2. FEBS Lett. 2012;586(16):2375-81. https://doi.org/1 0.1016/j.febslet.2012.05.049.

19. Li D, Shan W, Fang Y, Wang P, Li J. MiR-137 acts as a tumor suppressor via inhibiting CXCL12 in human glioblastoma. Oncotarget. 2017:8(60):10126270. https://doi.org/10.18632/oncotarget.20589.

20. Luo M, Wu L, Zhang K, Wang H, Zhang T, Gutierrez L, et al. MiR-137 regulates ferroptosis by targeting glutamine transporter SLC1A5 in melanoma. Cell Death Differ. 2018;25(8):1457-72. https://doi.org/10.1038/ s41418-017-0053-8

21. Qin Y, Zhang S, Deng S, An G, Qin X, Li F, et al. Epigenetic silencing of miR137 induces drug resistance and chromosomal instability by targeting AURKA in multiple myeloma. Leukemia. 2017;31(5):1123-35. https://doi. org/10.1038/leu.2016.325.

22. Liu X, Chen L, Tian XD, Zhang T. MiR-137 and its target TGFA modulate cell growth and tumorigenesis of non-small cell lung cancer. Eur Rev Med Pharmacol Sci. 2017;21(3):511-7.

23. Zhang W, Chen JH, Shan T, Aguilera-Barrantes I, Wang LS, Huang TH, et al. MiR-137 is a tumor suppressor in endometrial cancer and is repressed by DNA hypermethylation. Lab Investig. 2018;98(11):1397-407. https://doi.org/1 0.1038/s41374-018-0092-x.

24. Jiang K, Ren C, Nair VD. MicroRNA-137 represses KIf4 and Tbx3 during differentiation of mouse embryonic stem cells. Stem Cell Res. 2013;11(3): 1299-313. https://doi.org/10.1016/j.scr.2013.09.001.

25. Silber J, Lim DA, Petritsch C, Persson Al, Maunake AK, Yu M, et al. MiR-124 and miR-137 inhibit proliferation of glioblastoma multiforme cells and induce differentiation of brain tumor stem cells. BMC Med. 2008;6(1):14. https://doi.org/10.1186/1741-7015-6-14.

26. Sun GQ, Ye P, Murai K, Lang MF, Li S, Zhang H, et al. MiR-137 forms a regulatory loop with nuclear receptor TLX and LSD1 in neural stem cells. Nat Commun. 2011;2(1):529. https://doi.org/10.1038/ncomms1532.

27. Szulwach KE, Li X, Smrt RD, Li Y, Luo Y, Lin L, et al. Cross talk between microRNA and epigenetic regulation in adult neurogenesis. J Cell Biol. 2010; 189(1):127-41. https://doi.org/10.1083/jcb.200908151.
28. Tarantino C, Paolella G, Cozzuto L, Minopoli G, Pastore L, Parisi S, et al. MiRNA 34a, 100, and 137 modulate differentiation of mouse embryonic stem cells. FASEB J. 2010;24(9):3255-63. https://doi.org/10.1096/fj.09-152207.

29. Crowley JJ, Collins AL, Lee RJ, Nonneman RJ, Farrell MS, Ancalade N, et al. Disruption of the microRNA 137 primary transcript results in early embryonic lethality in mice. Biol Psychiatry. 2015;77(2):e5-7. https://doi. org/10.1016/j.biopsych.2014.05.022.

30. Hollins SL, Goldie BJ, Carroll AP, Mason EA, Walker FR, Eyles DW, et al. Ontogeny of small RNA in the regulation of mammalian brain development. BMC Genomics. 2014;15(1):777. https://doi.org/10.1186/14 71-2164-15-777.

31. Smrt RD, Szulwach KE, Pfeiffer RL, Li X, Guo W, Pathania M, et al. MicroRNA miR-137 regulates neuronal maturation by targeting ubiquitin ligase mind bomb-1. Stem Cells. 2010;28(6):1060-70. https://doi.org/10.1002/stem.431.

32. Ma X, Fan C, Wang Y, Du Y, Zhu Y, Liu H, et al. MiR-137 knockdown promotes the osteogenic differentiation of human adipose-derived stem cells via the LSD1/BMP2/SMAD4 signaling network. J Cell Physiol. 2020; 235(2):909-19. https://doi.org/10.1002/jcp.29006.

33. Canalis E, Adams DJ, Boskey A, Parker K, Kranz L, Zanotti S. Notch signaling in osteocytes differentially regulates cancellous and cortical bone remodeling. J Biol Chem. 2013;288(35):25614-25. https://doi.org/10.1074/jbc. M113.470492.

34. Engin F, Yao Z, Yang T, Zhou G, Bertin T, Jiang MM, et al. Dimorphic effects of Notch signaling in bone homeostasis. Nat Med. 2008;14(3):299-305. https://doi.org/10.1038/nm1712.

35. Zanotti S, Canalis E. Notch signaling and the skeleton. Endocr Rev. 2016; 37(3):223-53. https://doi.org/10.1210/er.2016-1002.

36. Jarriault S, Brou C, Logeat F, Schroeter EH, Kopan R, Israel A. Signalling downstream of activated mammalian Notch. Nature. 1995;377(6547):355-8. https://doi.org/10.1038/377355a0.

37. Shen $\mathrm{Q}$, Christakos S. The vitamin D receptor, Runx2, and the Notch signaling pathway cooperate in the transcriptional regulation of osteopontin. J Biol Chem. 2005;280(49):40589-98. https://doi.org/10.1074/ jbc.M504166200.

38. Zhang Y, Lian JB, Stein JL, van Wijnen AJ, Stein GS. The Notch-responsive transcription factor Hes-1 attenuates osteocalcin promoter activity in osteoblastic cells. J Cell Biochem. 2009;108(3):651-9. https://doi.org/10.1002/ jcb.22299.

39. Zanotti S, Smerdel-Ramoya A, Canalis E. HES1 (hairy and enhancer of split 1) is a determinant of bone mass. J Biol Chem. 2011;286(4):2648-57. https:// doi.org/10.1074/jbc.M110.183038.

40. Han F, Wang S, Chang Y, Li C, Yang J, Han Z, et al. Triptolide prevents extracellular matrix accumulation in experimental diabetic kidney disease by targeting microRNA-137/Notch1 pathway. J Cell Physiol. 2018;233(3):222537. https://doi.org/10.1002/jcp.26092.

41. Li H, Zhu Z, Liu J, Wang J, Qu C. MicroRNA-137 regulates hypoxia-induced retinal ganglion cell apoptosis through Notch1. Int J Mol Med. 2018;41(3): 1774-82. https://doi.org/10.3892/ijmm.2017.3319.

42. Shi F, Dong Z, Li H, Liu X, Liu H, Dong R. MicroRNA-137 protects neurons against ischemia/reperfusion injury through regulation of the Notch signaling pathway. Exp Cell Res. 2017;352(1):1-8. https://doi.org/10.1016/j. yexcr.2017.01.015

43. Wang $X$, Zhang $G$, Cheng $Z$, Dai $L$, Jia $L$, Jing $X$, et al. Knockdown of IncRNAXIST suppresses proliferation and TGF- $\beta 1$-induced EMT in NSCLC through the Notch-1 pathway by regulation of miR-137. Genet Test Mol Biomarkers. 2018;22(6):333-42. https://doi.org/10.1089/gtmb.2018.0026.

44. Zeng JS, Zhang ZD, Pei L, Bai ZZ, Yang Y, Yang H, et al. CBX4 exhibits oncogenic activities in breast cancer via Notch1 signaling. Int J Biochem Cell Biol. 2018;95:1-8. https://doi.org/10.1016/j.biocel.2017.12.006.

45. Augert A, Eastwood E, Ibrahim AH, Wu N, Grunblatt E, Basom R, et al. Targeting NOTCH activation in small cell lung cancer through LSD1 inhibition. Sci Signal. 2019;12(567):eaau2922.

46. Viale-Bouroncle $\mathrm{S}$, Gosau M, Morsczeck C. NOTCH1 signaling regulates the BMP2/DLX-3 directed osteogenic differentiation of dental follicle cells. Biochem Biophys Res Commun. 2014;443(2):500-4. https://doi.org/10.1016/j. bbrc.2013.11.120.

47. Wang D, Zeng Q, Song R, Ao L, Fullerton DA, Meng X. Ligation of ICAM-1 on human aortic valve interstitial cells induces the osteogenic response: critical role of the Notch1-NF-kB pathway in BMP-2 expression. Biochim Biophys Acta. 2014;1843(11):2744-53. https://doi.org/10.1016/j.bbamcr.2014. 07.017 . 
48. Sun J, Ermann J, Niu N, Yan G, Yang Y, Shi Y, et al. Histone demethylase LSD1 regulates bone mass by controlling WNT7B and BMP2 signaling in osteoblasts. Bone Res. 2018;6(1):14. https://doi.org/10.1038/s41413-018-0015-x.

49. Yang $C$, Wang $W$, Liang JX, Li G, Vellaisamy K, Wong CY, et al. A rhodium (III)-based inhibitor of lysine-specific histone demethylase 1 as an epigenetic modulator in prostate cancer cells. J Med Chem. 2017;60(6):2597-603. https://doi.org/10.1021/acs.jmedchem.7b00133.

50. Kong L, Zuo R, Wang M, Wang W, Xu J, Chai Y, et al. Silencing microRNA-137$3 p$, which targets RUNX2 and CXCL12 prevents steroid-induced osteonecrosis of the femoral head by facilitating osteogenesis and angiogenesis. Int I Biol Sci. 2020;16(4):655-70. https://doi.org/10.7150/ijbs.38713.

51. Althoff K, Beckers A, Odersky A, Mestdagh P, Köster J, Bray IM, et al. MiR-137 functions as a tumor suppressor in neuroblastoma by downregulating KDM1 A. Int J Cancer. 2013;133(5):1064-73. https://doi.org/10.1002/ijc.28091.

52. Chen L, Wang X, Huang W, Ying T, Chen M, Cao J, et al. MicroRNA-137 and its downstream target LSD1 inversely regulate anesthetics-induced neurotoxicity in dorsal root ganglion neurons. Brain Res Bull. 2017;135:1-7. https://doi.org/10.1016/j.brainresbull.2017.09.004.

53. Hartenstein AY, Rugendorff A, Tepass U, Hartenstein $V$. The function of the neurogenic genes during epithelial development in the drosophila embryo. Development. 1992;116(4):1203-20. https://doi.org/10.1242/dev.116.4.1203.

54. Miele L, Osborne B. Arbiter of differentiation and death: Notch signaling meets apoptosis. J Cell Physiol. 1999;181(3):393-409. https://doi.org/10.1002/ (SICI) 1097-4652(199912)181:3<393::AID-JCP3>3.0.CO;2-6.

55. Ongaro A, Pellati A, Bagheri L, Rizzo P, Caliceti C, Massari L, et al. Characterization of Notch signaling during osteogenic differentiation in human osteosarcoma cell line MG63. J Cell Physiol. 2016;231(12):2652-63. https://doi.org/10.1002/jcp.25366.

56. de la Pompa JL, Wakeham A, Correia KM, Samper E, Brown S, Aguilera RJ, et al. Conservation of the Notch signalling pathway in mammalian neurogenesis. Development. 1997;124(6):1139-48. https://doi.org/10.1242/ dev.124.6.1139.

57. Leimeister C, Dale K, Fischer A, Klamt B. Hrabe de Angelis M, Radtke F, et al. Oscillating expression of $\mathrm{c}-\mathrm{Hey} 2$ in the presomitic mesoderm suggests that the segmentation clock may use combinatorial signaling through multiple interacting bHLH factors. Dev Biol. 2000;227(1):91-103. https://doi.org/10.1 006/dbio.2000.9884.

58. Leimeister C, Schumacher N, Steidl C, Gessler M. Analysis of Heyl expression in wild-type and Notch pathway mutant mouse embryos. Mech Dev. 2000; 98(1-2):175-8. https://doi.org/10.1016/50925-4773(00)00459-7.

59. Di Stefano L, Walker JA, Burgio G, Corona DF, Mulligan P, Näär AM, et al. Functional antagonism between histone H3K4 demethylases in vivo. Genes Dev. 2011;25(1):17-28. https://doi.org/10.1101/gad.1983711.

60. Han X, Ranganathan P, Tzimas C, Weaver KL, Jin K, Astudillo L, et al. Notch represses transcription by PRC2 recruitment to the ternary complex. Mol Cancer Res. 2017; 15(9):1173-83. https:/doi.org/10.1158/1541-7786.MCR-17-0241.

61. Lopez Cl, Saud KE, Aguilar R, Berndt FA, Cánovas J, Montecino M, et al. The chromatin modifying complex CoREST/LSD1 negatively regulates notch pathway during cerebral cortex development. Dev Neurobiol. 2016;76(12): 1360-73. https://doi.org/10.1002/dneu.22397.

62. Mulligan P, Yang F, Di Stefano L, Ji JY, Ouyang J, Nishikawa JL, et al. A SIRT1-LSD1 corepressor complex regulates Notch target gene expression and development. Mol Cell. 2011;42(5):689-99. https://doi.org/10.1016/j. molcel.2011.04.020.

63. Xu T, Park SS, Giaimo BD, Hall D, Ferrante F, Ho DM, et al. RBPJ/CBF1 interacts with L3MBTL3/MBT1 to promote repression of Notch signaling via histone demethylase KDM1A/LSD1. EMBO J. 2017:36(21):3232-49. https:// doi.org/10.15252/embj.201796525.

64. Yatim A, Benne C, Sobhian B, Laurent-Chabalier S, Deas O, Judde JG, et al. NOTCH1 nuclear interactome reveals key regulators of its transcriptional activity and oncogenic function. Mol Cell. 2012;48(3):445-58. https://doi. org/10.1016/j.molcel.2012.08.022.

\section{Publisher's Note}

Springer Nature remains neutral with regard to jurisdictional claims in published maps and institutional affiliations.

\section{Ready to submit your research? Choose BMC and benefit from}

- fast, convenient online submission

- thorough peer review by experienced researchers in your field

- rapid publication on acceptance

- support for research data, including large and complex data types

- gold Open Access which fosters wider collaboration and increased citations

- maximum visibility for your research: over $100 \mathrm{M}$ website views per year

At BMC, research is always in progress.

Learn more biomedcentral.com/submissions 